\title{
Three-Dimensional Simulation of Stress Dependent Thermal Oxidation
}

\author{
Ch. Hollauer, H. Ceric, and S. Selberherr \\ Institute for Microelectronics, TU Vienna, Gußhausstraße 27-29/E360, 1040 Wien, Austria \\ Phone: +43-1-58801/36036, Fax: +43-1-58801/36099, E-mail: hollauer@iue.tuwien.ac.at
}

\begin{abstract}
We present a sophisticated numerical model for the simulation of thermal oxidation on complex three-dimensional structures. This comprehensive oxidation model takes into account that the diffusion of oxidants, the chemical reaction, and the volume increase occur simultaneously in a so-called reactive layer which has a spatial finite width, in contrast to the sharp interface between silicon and dioxide in the conventional formulation. Our oxidation model also includes the coupled stress dependence of the oxidation process, because the influence of stress is shown to be considerable.
\end{abstract}

\section{INTRODUCTION}

If a surface of a silicon body has contact with an oxidizing atmosphere, the chemical reaction of oxidants with silicon ( $\mathrm{Si}$ ) forms silicon dioxide $\left(\mathrm{SiO}_{2}\right)$. Furthermore, the oxidants diffuse through an already existing $\mathrm{SiO}_{2}$-domain to the $\mathrm{Si}-\mathrm{SiO}_{2}$ interface [1] where the chemical reaction of the oxidants with silicon takes place.

The parts of silicon which should not be oxidized are masked by a layer of silicon nitride $\left(\mathrm{Si}_{3} \mathrm{Ni}_{4}\right)$, because $\mathrm{Si}_{3} \mathrm{Ni}_{4}$ prevents the oxidant diffusion on the subjacent $\mathrm{SiO}_{2}$-layer. During the oxidation process the chemical reaction consumes $\mathrm{Si}$ and the newly formed $\mathrm{SiO}_{2}$ has more than twice the volume of the original $\mathrm{Si}$. This significant volume increase leads to large displacements in the materials.

If the additional volume is prevented from expanding as desired, e.g. by the $\mathrm{Si}_{3} \mathrm{Ni}_{4}$ - mask, mechanical stresses arise in the materials. Since there is a strong stress dependence of the oxidant diffusion and the chemical reaction, the oxidation process itself is considerably influenced by stress.

So thermal oxidation is a complex process where the three subprocesses oxidant diffusion, chemical reaction, and volume increase occur simultaneously. From a mathematical point of view the oxidation process can be described by a coupled system of partial differential equations, one for the diffusion of oxidants through $\mathrm{SiO}_{2}$, the second for the conversion of $\mathrm{Si}$ into $\mathrm{SiO}_{2}$ at the interface, and a third for the mechanical problem of the complete oxidized structure. The whole mathematical formulation is numerically solved by applying the finite element method.

The oxidation rate mainly depends on the oxidizing ambient, especially on the temperature, the pressure, and the oxidant species. For practical use of an oxidation model it is important to carefully control the ambient parameters of the oxidation process. Therefore in our model these parameters, e.g the temperature profile, are adjustable easily.

\section{THE MODEL}

In our oxidation model [2] we use a normalized silicon concentration $\eta(\vec{x}, t)$ [3] so that the value of $\eta$ is 1 in pure $\mathrm{Si}$ and 0 in pure $\mathrm{SiO}_{2}$. Advantageously our model takes into account that the diffusion of oxidants, the chemical reaction and the volume increase occur simultaneously in a so-called reaction layer. In contrast to the sharp interface between $\mathrm{Si}$ and $\mathrm{SiO}_{2}$ like in the standard model [4], this reaction layer has a spatial finite width (see Figure 3 ) where the value of $\eta$ lies between 0 and 1 .

Most of the other oxidation models [5][6] which are based on the Deal-Grove model [4], describe the $\mathrm{SiO}_{2}$-growth more or less by a moving boundary problem. Unfortunately the handling of moving boundary problems becomes very complicated for complex three-dimensional structures [7].

Our oxidation model with the normalized silicon concentration and the reaction layer always exhibits the same reliability independent of the three-dimensional geometry. Additionally also thin film or dry oxidation [8] is properly treated by our model without any modification.

\section{A. Oxidant Diffusion}

The diffusion of oxidants is described by

$$
D(p) \Delta C(\vec{x}, t)=k(\eta, p) C(\vec{x}, t),
$$

where $\Delta$ is the Laplace operator, $C(\vec{x}, t)$ is the oxidant concentration, and $D(p)$ is the stress dependent diffusion coefficient:

$$
D(p)=D_{0} \exp \left(-\frac{p V_{D}}{k_{B} T}\right)
$$

Here $D_{0}$ is the low stress diffusion coefficient, $p$ is the hydrostatic pressure in the respective material, $V_{D}$ is the activation volume, $k_{B}$ is the Boltzmann's constant, and $T$ is the temperature in Kelvin.

$k(\eta, p)$ is the stress dependent strength of a spatial sink and not just a reaction coefficient at a sharp interface:

$$
k(\eta, p)=\eta(\vec{x}, t) k_{\text {max }} \exp \left(-\frac{p V_{k}}{k_{B} T}\right) .
$$

In (3) we define that $k(\eta, p)$ is linearly proportional to $\eta(\vec{x}, t)$. Furthermore (2) and (3) are only valid for a pressure $p \geq 0$.

\section{B. Dynamics of $\eta$}

The dynamics of $\eta$ is described by

$$
\frac{\partial \eta(\vec{x}, t)}{\partial t}=-\frac{1}{\lambda} k(\eta, p) C(\vec{x}, t) / N_{1},
$$

where $\lambda$ is the volume expansion factor $(=2.25)$ for the reaction from $\mathrm{Si}$ to $\mathrm{SiO}_{2}$, and $N_{1}$ is the number of oxidant molecules incorporated into one unit volume of $\mathrm{SiO}_{2}$. 


\section{Mechanics}

The chemical reaction of silicon and oxygen causes a significant volume increase. The normalized additional volume after time $\Delta t$ is

$$
V_{r e l}^{a d d}=\frac{\lambda-1}{\lambda} \Delta t k(\eta, p) C(\vec{x}, t) / N_{1} .
$$

The mechanical problem is described by the equilibrium relations of the stress components

$$
\begin{gathered}
\frac{\partial \sigma_{x x}}{\partial x}+\frac{\partial \sigma_{x y}}{\partial y}+\frac{\partial \sigma_{x z}}{\partial z}=0 \\
\frac{\partial \sigma_{y x}}{\partial x}+\frac{\partial \sigma_{y y}}{\partial y}+\frac{\partial \sigma_{y z}}{\partial z}=0 \\
\frac{\partial \sigma_{z x}}{\partial x}+\frac{\partial \sigma_{z y}}{\partial y}+\frac{\partial \sigma_{z z}}{\partial z}=0
\end{gathered}
$$

where the stress tensor $\tilde{\sigma}$ is given by

$$
\tilde{\sigma}=\mathbf{D}\left(\tilde{\varepsilon}-\tilde{\varepsilon_{0}}\right)+\tilde{\sigma_{0}} .
$$

Here $\mathbf{D}$ is the so-called material matrix which depends in the elastic case only on the Young's modulus E and the Poisson's ratio $\nu$, and in the viscoelastic case additionally on the Maxwellian relaxation time $\tau$ [9]. Furthermore, $\tilde{\varepsilon}$ is the strain tensor, $\tilde{\varepsilon_{0}}$ is the residual strain tensor, and $\tilde{\sigma_{0}}$ is the residual stress tensor.

The components $\varepsilon_{0, i i}(i$ stands for $x, y$ or $z$ ) of the residual strain tensor $\tilde{\varepsilon_{0}}$ are linear proportional to the normalized additional volume

$$
\varepsilon_{0, i i}=\frac{1}{3} V_{r e l}^{a d d}
$$

After discretization of the continuum, we obtain a linear equation system for the mechanical problem

$$
\mathbf{K} \vec{d}=\vec{f} \quad \text { with } \quad \mathbf{K}=\int_{\mathcal{V}} \mathbf{B}^{\mathbf{T}} \mathbf{D} \mathbf{B} d V,
$$

where $\mathbf{K}$ is the so-called stiffness matrix, $\vec{d}$ is the displacement vector, $\vec{f}$ is the force vector, and $\mathbf{B}$ is a discretized partial derivative matrix.

The force vector on a finite element depends on the residual strain tensor and thus also on the volume increase

$$
\vec{f}=\int_{\mathcal{V}} \mathbf{B}^{\mathbf{T}} \mathbf{D} \tilde{\varepsilon}_{0} d V .
$$

After solving the linear equation system (9) we obtain the displacement vector. Since the strain is the first derivative with respect to displacement

$$
\tilde{\varepsilon}=\mathbf{B} \vec{d}
$$

the stress can be determined with equation (7).

With the stress tensor the pressure for (2) and (3) can be determined by using the formula

$$
p=-\frac{\operatorname{Trace}(\tilde{\sigma})}{3}=-\frac{\sigma_{x x}+\sigma_{y y}+\sigma_{z z}}{3} .
$$

\section{SIMULATION PROCEDURE}

For the simulation procedure we perform a finite element discretization by splitting the three-dimensional structure into tetrahedral elements. In the next step we set the initial values for the oxidant concentration $C$ and the normalized silicon concentration $\eta$ on the grid nodes. E.g. $\eta$ is 1 in pure $\mathrm{Si}$.

As shown in Figure 1, we iterate over all finite elements and build the local equation system for each element for every actual discrete time. The local equation system describes the oxidation process numerically for one element. In order to describe the oxidation process on the complete simulation domain we need a global coupled equation system. The components of the global equation system are assembled from the local equation system by using the superposition principle.

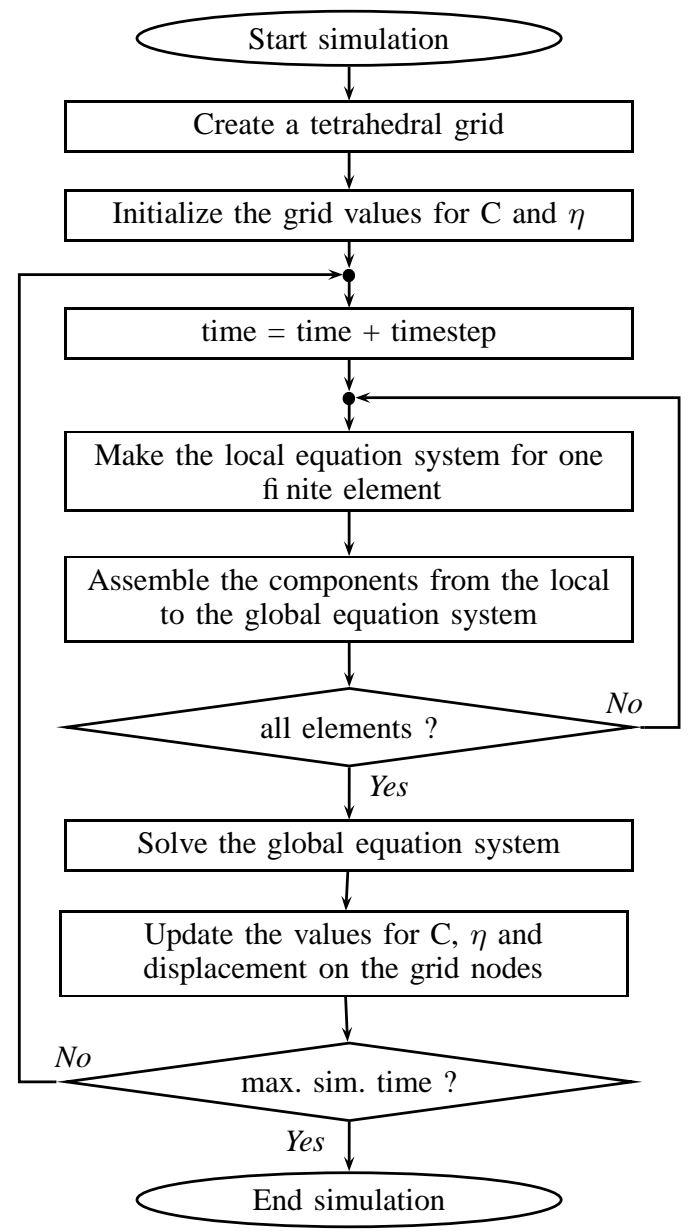

Figure 1: Simulation procedure

After the iteration over all elements is finished, the global assembled equation system is also completed. Now the global non-linear equation system can be solved, and we obtain the results for $C, \eta$, and the displacement values for the actual time step.

With these results we update the values for $C, \eta$, and the displacements for the actual time step, such that these values are always keeping pace with the actual simulation time. The displacement vector enables the calculation of the strain tensor (11) as well as the stress tensor (7). 


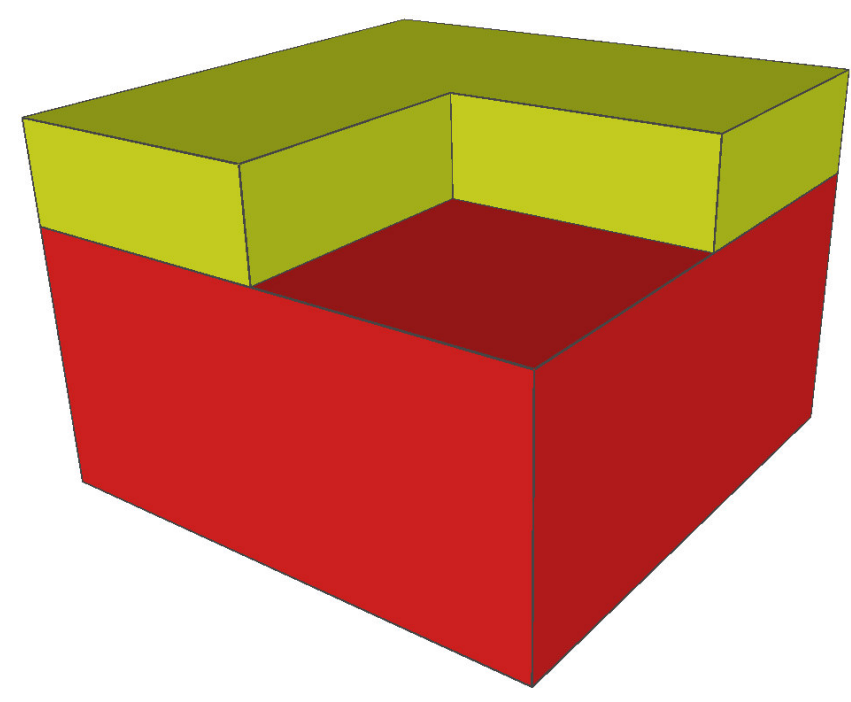

Figure 2: Initial structure of the $\mathrm{Si}-\mathrm{Si}_{3} \mathrm{Ni}_{4}$ - body before thermal oxidation

\section{REPRESENTATIVE EXAMPLE}

\section{A. Initial Structure}

We apply our oxidation model to the three-dimensional structure with $(0.8 \times 0.8) \mu \mathrm{m}$ floor space, as displayed on Figure 2. In this example the upper layer is a $0.15 \mu \mathrm{m}$ thick $\mathrm{L}$ - shaped $\mathrm{Si}_{3} \mathrm{Ni}_{4}$ - mask which prevents the oxidant diffusion on the subjacent $\mathrm{Si}$ - layer. Here the bottom surface is fixed and on the upper surface a free mechanical boundary condition is applied.

\section{B. Simulation Results}

The result of the oxidation process of the whole structure after a time $t_{1}$ is shown in Figure 3. Due to the $\mathrm{L}$ - shaped mask here the effect of the three-dimensional oxidation process is pronounced, because the shape of the $\mathrm{SiO}_{2}$-region and the deformations are not continuous in any direction.

For a more physical interpretation of the simulation results with a sharp interface between $\mathrm{Si}$ and $\mathrm{SiO}_{2}$ the two regions can be extracted from the $\eta$-distribution by determining that $\eta \leq 0.5$ is $\mathrm{SiO}_{2}$ and $\eta>0.5$ is $\mathrm{Si}$ as shown in Figure IV-B. For an optimal comparison of the geometry before and after oxidation as well as the influence of stress, Figures 2-IV-B have the same perspectives and the same proportions.

\section{Stress Dependence}

In order to demonstrate the importance of the stress dependence we compare the results with and without the impact of stress. Since the oxidant diffusion and the chemical reaction are exponentially reduced with the pressure in the material, the oxidation process itself is highly stress dependent.

As shown in Figure IV-B the highest pressure in $\mathrm{SiO}_{2}$ is under the edge of the $\mathrm{Si}_{3} \mathrm{Ni}_{4}$ - mask, because in this area the stiffness of the mask prevents the desired volume expansion of the newly formed $\mathrm{SiO}_{2}$. Due to the mentioned stress dependence the oxidation rate in these areas is considerably reduced (see Figure IV-B).

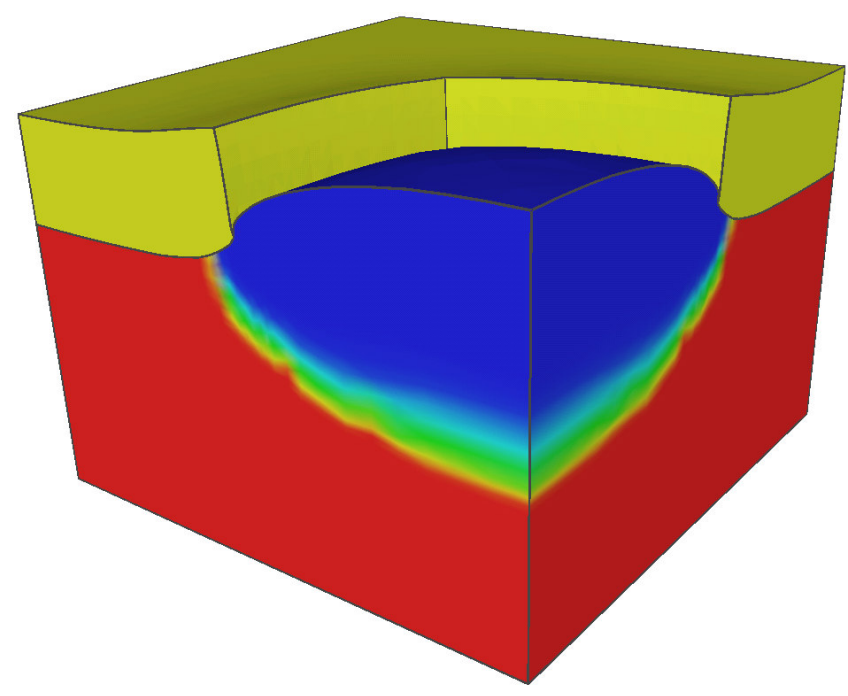

Figure 3: $\eta$-distribution and reaction layer after thermal oxidation at time $t_{1}$

If the stress dependence is not included in the simulation of the oxidation process, the simulation results do not agree with the real physical behavior, because the oxidant diffusion and the chemical reaction also occur under the $\mathrm{Si}_{3} \mathrm{Ni}_{4}$ - mask without restriction. Because of this phenomenon the $\mathrm{SiO}_{2}$ - region at the same oxidation conditions is much more expanded than with the stress dependence as shown in Figure 4. In addition, the larger forces under the $\mathrm{Si}_{3} \mathrm{Ni}_{4}$ - mask, which result from the larger pressure domain (see Figure 5) in this area, cause larger displacements of the mask.

The stiffness of the $\mathrm{Si}_{3} \mathrm{Ni}_{4}$ - mask is approximately six times larger than the stiffness of $\mathrm{SiO}_{2}$ and so the displacements in $\mathrm{SiO}_{2}$ are also much more larger than in the $\mathrm{Si}_{3} \mathrm{Ni}_{4}$ - mask which leads to the well-known bird's beak effect.

\section{SUMMARY AND CONCLUSION}

An oxidation model which takes the real physical behavior of the whole oxidation process under full control of the ambient parameters into account, has been presented. Our model is based on a normalized silicon concentration and a reaction layer with a spatial finite width in contrast to the moving boundary concept and a sharp $\mathrm{Si}-\mathrm{SiO}_{2}$ - interface in the conventional formulation.

The reaction layer takes into account that the diffusion of oxidants, the chemical reaction and the volume increase occur simultaneously. In contrast to the moving boundary concept, our normalized silicon concentration concept also works on complex three-dimensional structures without restriction.

For a physical interpretation with a sharp interface between $\mathrm{Si}$ and $\mathrm{SiO}_{2}$ the two regions can be extracted from the normalized silicon distribution by determining that a value equal or less 0.5 is $\mathrm{SiO}_{2}$ and a value larger than 0.5 is $\mathrm{Si}$. Furthermore thick film as well as thin film oxidation are properly treated without a need of modification.

For the mechanical behavior of the materials an elastic or viscoelastic model can be applied because the materials 


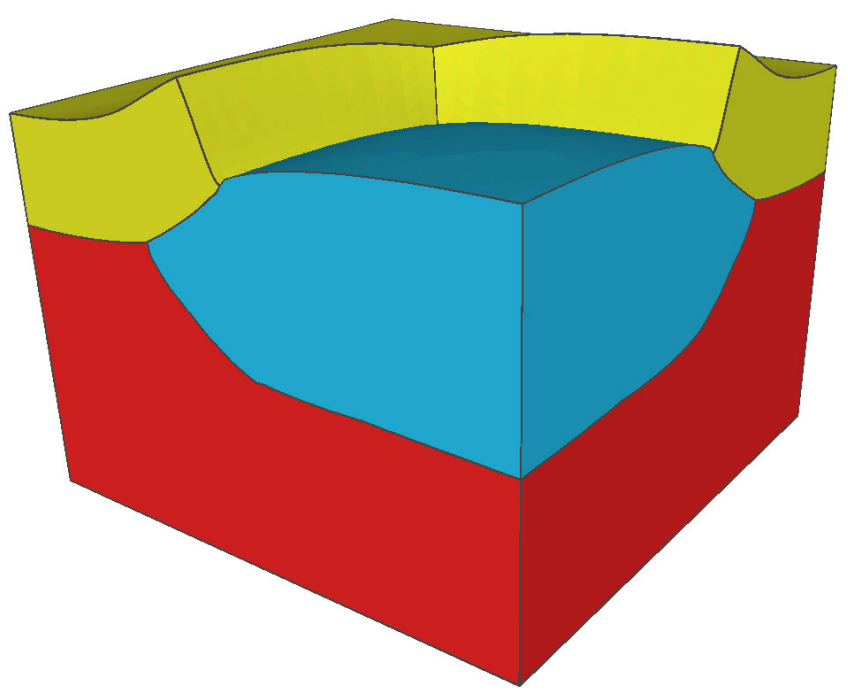

Figure 4: $\mathrm{SiO}_{2}$-region (sharp interface) without stress dependent oxidation at time $t_{1}$.

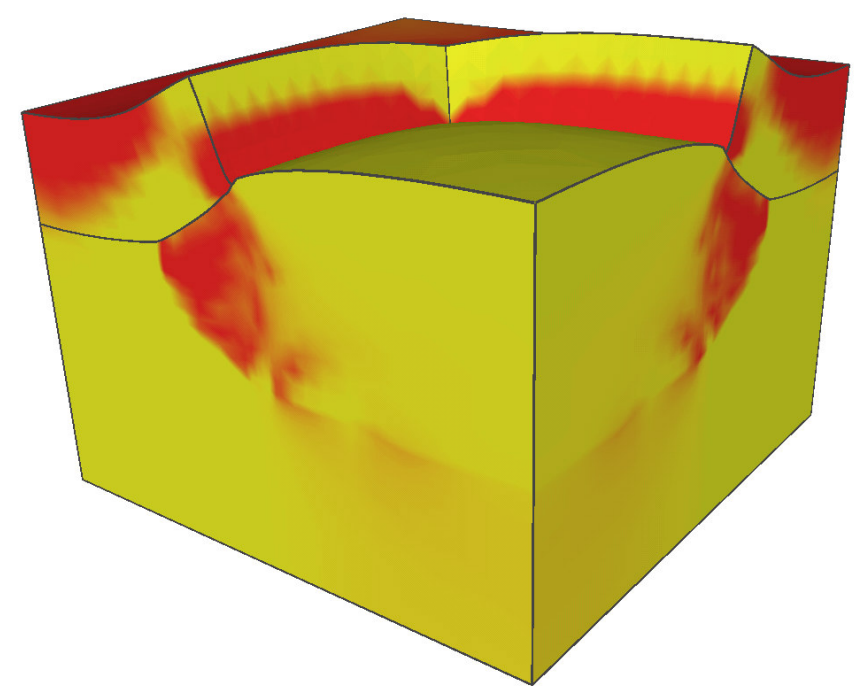

Figure 5: Pressure distribution without stress dependent oxidation at time $t_{1}$.

$\mathrm{SiO}_{2}$ and $\mathrm{Si}_{3} \mathrm{Ni}_{4}$ have a viscoelastic behavior [10]. In the viscoelastic mechanics model we use a Maxwellian formulation. The whole mathematical formulation of the oxidation process, which is described by a coupled system of partial differential equations, is solved by applying the finite element method.

The important influence of stress on thermal oxidation was investigated. It was shown that the highest hydrostatic pressure in $\mathrm{SiO}_{2}$ is under the edge of the $\mathrm{Si}_{3} \mathrm{Ni}_{4}$-mask and that the results agree only for stress dependent oxidation with the real physical behavior. As a result of the strong stress dependence of the oxidant diffusion and the chemical reaction the oxidation rate in this area is considerably reduced.

If the stress is not taken into account the oxidation process also occurs under the $\mathrm{Si}_{3} \mathrm{Ni}_{4}$-mask without restriction and so the $\mathrm{SiO}_{2}$-domain and the displacements are predicted too large in relation to the real physical process.

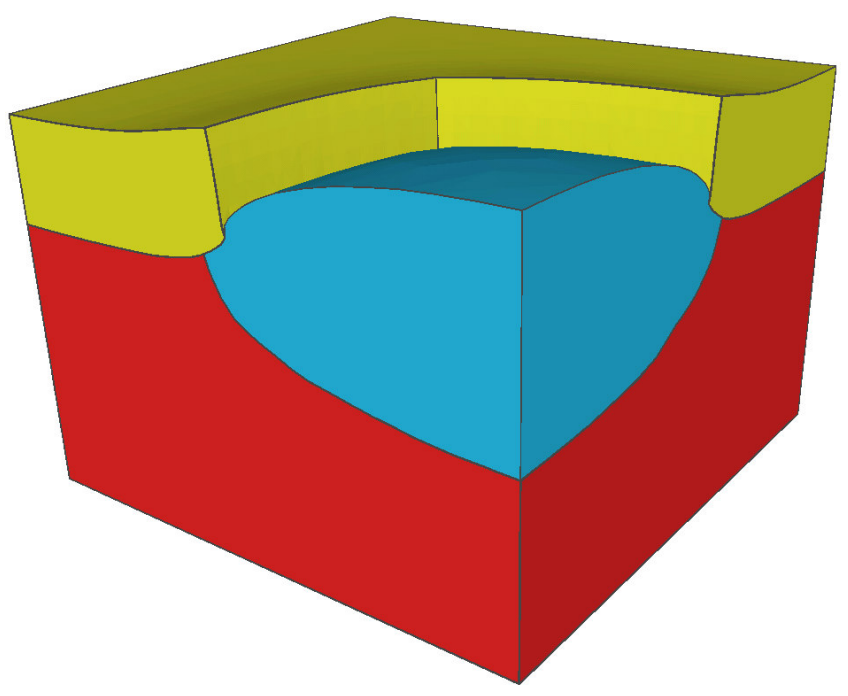

Figure 6: $\mathrm{SiO}_{2}$ - region (sharp interface) with stress dependent oxidation at time $t_{1}$.

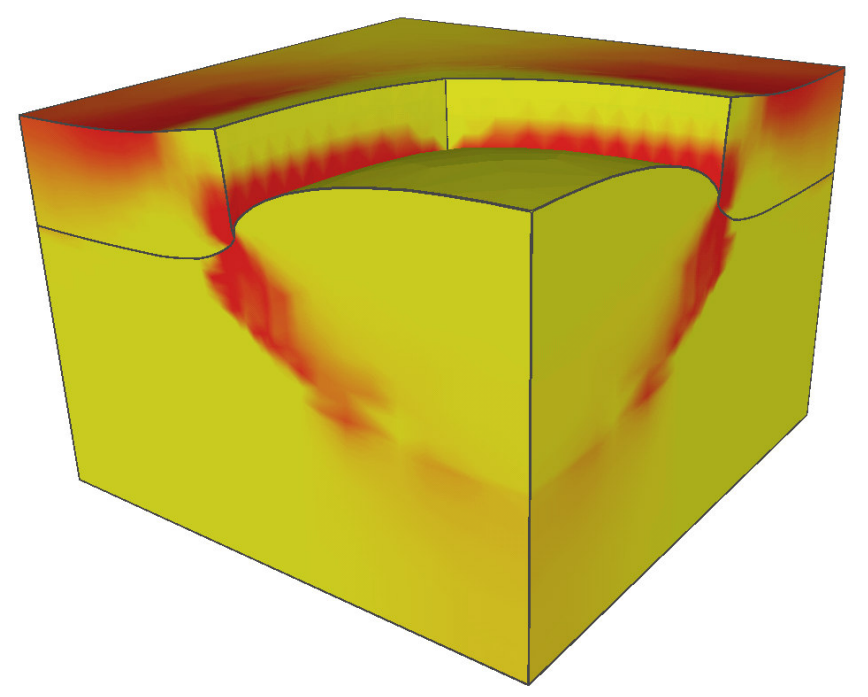

Figure 7: Pressure distribution with stress dependent oxidation at time $t_{1}$.

\section{REFERENCES}

[1] D. R. Hamann, Phys. Rev. Lett. 81, 3447 (1998).

[2] C. Hollauer, H. Ceric, and S. Selberherr, in Proc. ESSDERC 2003 (Estoril, Portugal, 2003), pp. 383-386.

[3] E. Rank and U. Weinert, IEEE Trans. CAD 9, 543 (1990).

[4] B. Deal and A. Grove, J. Appl. Phys. 36, 3770 (1965).

[5] A. Poncet, IEEE Trans. CAD 4, 41 (1985).

[6] S. Cea, Ph.D. thesis, University of Florida, USA, 1996.

[7] V. Senez, S. Bozek, and B. Baccus, in Proc. IEDM 1996 (San Francisco, USA, 1996), pp. 705-708.

[8] H. Z. Massoud and J. D. Plummer, J. Appl. Phys. 62, 3416 (1987).

[9] V. Senez et al., IEEE Trans. Elect. Dev. 43, 720 (1996).

[10] H. Matsumoto and M. Fukuma, in Proc. IEDM 1983 (Washington, USA, 1983), pp. 39-43. 